\title{
炔丙醇与烯醇硅醚的直接亲核取代反应
}

\author{
李金玲刘会丽张顺吉* \\ (淮北师范大学化学与材料科学学院 安徽淮北 235000)
}

\begin{abstract}
摘要 报道了一种在对甲苯磺酸催化下炔丙醇和烯醇硅梄的直接亲核取代反应，并有效合成 $\gamma$-炔基酮类化合物的新方 法. 该反应在敞口环境下使用未经干燥处理的溶剂即可获得良好的产率.

关键词＼cjkstart炔丙醇；烯醇硅梄；亲核取代；催化；对甲苯磺酸
\end{abstract}

\section{Direct Nucleophilic Substitution of Propargyl Alcohols with Enoxysilanes}

\author{
Li, Xinling Liu, Huili Zhang, Shunji* \\ (Department of Chemistry, Huaibei Normal University, Huaibei, Anhui 235000)
}

\begin{abstract}
A novel and efficient synthesis of $\gamma$-alkynyl ketones by the direct nucleophilic substitution of propargyl alcohols with enoxysilanes in the presence of a catalytic amount of $p$-toluenesulfonic acid monohydrate, has been developed. The reactions can be performed in an undried solvent under an air atmosphere to obtain the desired products in good yields.

Keywords propargyl alcohol; enoxysilane; nucleophilic substitution; catalyzed; $p$-toluenesulfonic acid
\end{abstract}

\section{Introduction}

Propargyl alcohols, which possess inherent alkynyl and hydroxyl functional groups, are among the valuable bifunctional building blocks in organic synthesis. The simple generation and conversion of propargyl alcohols into various valuable products make them powerful and attractive materials in synthesis chemistry. ${ }^{[1]}$ In particular, nucleophilic substitution of the hydroxyl group of propargyl alcohols has been widely investigated for carbon-carbon and carbon-heteroatom bond formation. Unfortunately, the carbon-centered nucleophiles for the construction of $\mathrm{sp}^{3}-\mathrm{sp}^{3}-\mathrm{C}-\mathrm{C}$ bonds in the reaction have been limited to allyl silanes ${ }^{[2]}$ and 1,3-dicarbonyl derivatives. ${ }^{[3]}$ Enoxysilanes are known to be good nucleophiles in many reactions, but direct nucleophilic substitution of propargyl alcohols with enoxysilanes for $\mathrm{sp}^{3}-\mathrm{sp}^{3}-\mathrm{C}-\mathrm{C}$ bond formation has seldom been reported. ${ }^{[4]}$ In order to construct $\mathrm{sp}^{3}-\mathrm{sp}^{3}$ $\mathrm{C}-\mathrm{C}$ bonds, propargyl alcohols are usually transformed into propargylic acetates. Over the past few years, several methodologies for the substitution of propargylic acetates with enoxysilanes in the presence of metal catalysts such as iridium complexes $\left[\operatorname{Ir}(\mathrm{cod})\left\{(\mathrm{OPh})_{3}\right\}_{2}\right] \mathrm{OTf}{ }^{[5]}$ as well as $\mathrm{Cu}(\mathrm{OTf})_{2},{ }^{[6]} \mathrm{FeCl}_{3},{ }^{[7]}$ and $\mathrm{InCl}_{3}{ }^{[8]}$ have been reported. In 2015, Balamurugan et al. ${ }^{[9]}$ reported silver hexafluoroan- timonate $(\mathrm{V})\left(\mathrm{AgSbF}_{6}\right)$ serves as a catalyst for the coupling of propargylic alcohols and ketones to furnish $\gamma$-alkynyl ketones.

In this letter, we report our results for the direct nucleophilic substitution of propargyl alcohols with enoxysilanes catalyzed by $p$-toluenesulfonic acid monohydrate (PTSA) to afford the corresponding $\gamma$-alkynyl carbonyl compounds. This method does not require expensive catalysts, explosive solvents, or long reaction times. Furthermore, the reactions can be performed under mild conditions without special precautions to exclude moisture or air from the reaction system.

\section{Results and discussion}

Propargyl alcohol 1a was treated with enoxysilane 2a in acetonitrile at room temperature in the presence of a catalytic amount of iron(III) chloride, cuprous iodide, sulfuric acid, or trifluoroacetic acid. However, the desired product 3a was not detected. Poor reactivity was observed in the presence of $10 \mathrm{~mol} \%$ PTSA. The reaction remained incomplete even after being allowed to proceed overnight at room temperature and $\mathbf{3 a}$ was obtained in only $30 \%$ yield. Gratifyingly, when the temperature was raised to $60{ }^{\circ} \mathrm{C}$, 1a was completely consumed within 2 min, as confirmed by thin-layer chromatography (TLC) monitoring, and the

\footnotetext{
* Corresponding authors. E-mail: sjzhang@chnu.edu.cn

Received July 4, 2020; revised August 6, 2020; published online August 26, 2020.
} 
corresponding product was isolated in good yield (Table 1, Entry 1). The presence of excess enoxysilane is also crucial for the sufficient conversion of propargyl alcohol. The use of 4 equiv. of enoxysilane was essential for obtaining a high product yield. Thus, the optimal reaction conditions were finally established as $10 \mathrm{~mol} \%$ PTSA and 4 equiv of enoxysilane in acetonitrile at $60{ }^{\circ} \mathrm{C}$.

With the optimal conditions in hand, the scope of propargyl alcohols for this transformation was next evaluated. Both electron-donating and electron-withdrawing aromatic substrates reacted smoothly with $\mathbf{2 a}$, affording the corresponding alkylated products in high yields (Table 1, Entries $2 \sim 11$ ). Furthermore, it was found that the starting materials with electron-donating substituent $1 \mathbf{e} \sim \mathbf{1 k}$ reacted smoothly at room temperature. The experimental results suggest that the reaction proceeded via the formation of a propargylic cation intermediate, the stability of which made the substitution reaction more favorable. Functional groups, such as fluoro, chloro, bromo, benzyloxy, methyl, methoxy, and naphthyl groups did not affect the formation of $\mathrm{sp}^{3}-\mathrm{sp}^{3}-\mathrm{C}-\mathrm{C}$ bonds, but electron-withdrawing substrates required higher temperatures for reaction completion. The reaction proceeded successfully even when the alkyne substituent was changed from phenyl to $n$-pentyl (Table 1, Entry 7). The type of substituents on the propargyl carbon affects the susceptibility of propargyl alcohols to enoxysilanes. Coupling of benzylic propargylic alcohols with enoxysilane $\mathbf{2 a}$ was completed within 2 min furnishing the corresponding substitution products in good yields. However, the aliphatic propargyl alcohol $\mathbf{1 1}$ did not react with $2 \mathbf{a}$.

Based on these preliminary results, the scope of enoxy- silanes in this reaction was investigated. Gratifyingly, the reactions of enoxysilane $\mathbf{2 b}$ with propargyl alcohols $\mathbf{1 k}$ and 1f gave excellent yields of the expected products $3 \mathbf{l}$ and $\mathbf{3 m}$ in diastereomeric ratios $1.4: 1$ and $1.1: 1$, respectively (Table 1, Entries 13, 14). Other enoxysilanes 2c and 2d were also suitable for the present transformation. Propargyl alcohol $1 \mathbf{k}$ reacted with enoxysilanes $\mathbf{2 c}$ and $\mathbf{2 d}$ to afford 3n and 30 in $79 \%$ and $85 \%$ (Table 1, Entries 15, 16), respectively.

\section{Conclusions}

In conclusion, we have developed a simple strategy for the direct nucleophilic substitution of propargyl alcohols with enoxysilanes toward the construction of $\mathrm{sp}^{3}-\mathrm{sp}^{3}-\mathrm{C}-\mathrm{C}$ bonds. The reaction proceeds effectively under mild conditions in the presence of a simple, commercially available catalyst.

\section{Experimental section}

\subsection{General information}

All chemicals and solvents were used without further purification as commercially available. All manipulations were carried out under air atmosphere. Flash column chromatography was generally conducted on silica gel (200 300 mesh) and reactions were monitored by thin layer chromatography (TLC) using UV light to visualize the course of reactions. ${ }^{1} \mathrm{H}$ NMR and ${ }^{13} \mathrm{C}$ NMR spectra were measured on a Bruker spectrometer using $\mathrm{CDCl}_{3}$ as solvent. HRMS were performed on Agilent ESI-quadrupole.

Table 1 PTSA-catalyzed direct nucleophilic substitution of propargyl alcohols with enoxysilanes ${ }^{a}$

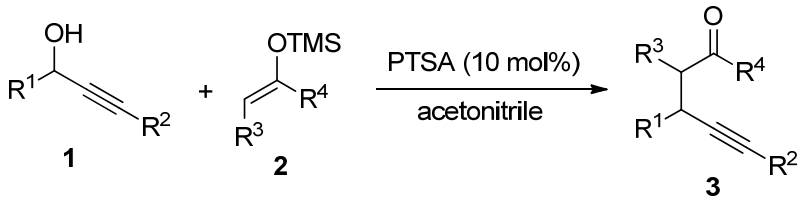

\begin{tabular}{|c|c|c|c|c|c|c|c|c|c|}
\hline Entry & 1 & $\mathrm{R}^{1}$ & $\mathrm{R}^{2}$ & 2 & $\mathrm{R}^{3}$ & $\mathrm{R}^{4}$ & $t /{ }^{\circ} \mathrm{C}$ & Product & Isolated yield $/ \%$ \\
\hline 1 & 1a & $\mathrm{Ph}$ & $\mathrm{Ph}$ & $2 a$ & $\mathrm{H}$ & COOMe & 60 & $3 a$ & 85 \\
\hline 2 & $1 b$ & $4-\mathrm{ClC}_{6} \mathrm{H}_{4}$ & $\mathrm{Ph}$ & $2 a$ & $\mathrm{H}$ & COOMe & 60 & $3 \mathbf{b}$ & 82 \\
\hline 3 & $1 \mathrm{c}$ & $4-\mathrm{BrC}_{6} \mathrm{H}_{4}$ & $\mathrm{Ph}$ & $2 a$ & $\mathrm{H}$ & COOMe & 60 & $3 c$ & 80 \\
\hline 4 & 1d & 2-MeO-5- $\mathrm{BrC}_{6} \mathrm{H}_{3}$ & $\mathrm{Ph}$ & $2 a$ & $\mathrm{H}$ & COOMe & 60 & 3d & 78 \\
\hline 5 & 1e & 2- $\mathrm{MeOC}_{6} \mathrm{H}_{4}$ & $\mathrm{Ph}$ & $2 a$ & $\mathrm{H}$ & COOMe & r.t. & $3 e$ & 83 \\
\hline 6 & 1f & $4-\mathrm{BnOC}_{6} \mathrm{H}_{4}$ & $\mathrm{Ph}$ & $2 a$ & $\mathrm{H}$ & COOMe & r.t. & $3 f$ & 90 \\
\hline 7 & $1 \mathrm{~g}$ & $4-\mathrm{BnOC}_{6} \mathrm{H}_{4}$ & $n$-Pentyl & $2 \mathbf{a}$ & $\mathrm{H}$ & COOMe & r.t. & $3 g$ & 88 \\
\hline 8 & $1 \mathrm{~h}$ & 2-Naphthyl & $\mathrm{Ph}$ & $2 \mathbf{a}$ & $\mathrm{H}$ & COOMe & r.t. & $3 \mathbf{h}$ & 91 \\
\hline 9 & $1 \mathbf{i}$ & 4-MeO-3- $-\mathrm{FC}_{6} \mathrm{H}_{3}$ & $\mathrm{Ph}$ & $\mathbf{2 a}$ & $\mathrm{H}$ & COOMe & r.t. & $3 \mathbf{i}$ & 88 \\
\hline 10 & $\mathbf{1 j}$ & $3,4-\mathrm{Me}_{2} \mathrm{C}_{6} \mathrm{H}_{3}$ & $\mathrm{Ph}$ & $\mathbf{2 a}$ & $\mathrm{H}$ & COOMe & r.t. & $3 \mathbf{j}$ & 92 \\
\hline 11 & $1 \mathrm{k}$ & $3,4-(\mathrm{MeO})_{2} \mathrm{C}_{6} \mathrm{H}_{3}$ & $\mathrm{Ph}$ & $\mathbf{2 a}$ & $\mathrm{H}$ & COOMe & r.t. & $3 \mathbf{k}$ & 95 \\
\hline 12 & 11 & $n-\mathrm{Bu}$ & $\mathrm{Ph}$ & $2 \mathbf{a}$ & $\mathrm{H}$ & COOMe & r.t. & - & 0 \\
\hline 13 & $1 \mathrm{k}$ & $3,4-(\mathrm{MeO})_{2} \mathrm{C}_{6} \mathrm{H}_{3}$ & $\mathrm{Ph}$ & $2 \mathbf{b}$ & & $-\left(\mathrm{CH}_{2}\right)_{4}-$ & r.t. & 31 & 93 \\
\hline 14 & 1f & $4-\mathrm{BnOC}_{6} \mathrm{H}_{4}$ & $\mathrm{Ph}$ & $2 \mathbf{b}$ & & $-\left(\mathrm{CH}_{2}\right)_{4}-$ & r.t. & $3 m$ & 95 \\
\hline 15 & $1 \mathrm{k}$ & $3,4-(\mathrm{MeO})_{2} \mathrm{C}_{6} \mathrm{H}_{3}$ & $\mathrm{Ph}$ & $2 \mathrm{c}$ & $\mathrm{H}$ & $\mathrm{Me}$ & r.t. & $3 n$ & 79 \\
\hline 16 & $1 \mathrm{k}$ & $3,4-(\mathrm{MeO})_{2} \mathrm{C}_{6} \mathrm{H}_{3}$ & $\mathrm{Ph}$ & 2d & $\mathrm{H}$ & $\mathrm{Ph}$ & r.t. & 30 & 85 \\
\hline
\end{tabular}

${ }^{a}$ Reaction conditions: propargyl alcohol $(0.2 \mathrm{mmol})$, enoxysilane $(0.8 \mathrm{mmol})$, PTSA $(0.02 \mathrm{mmol})$, acetonitrile $(2 \mathrm{~mL})$. 
Propargyl alcohols ${ }^{[10]} \mathbf{1 a} \sim \mathbf{1 j}$ and enoxysilanes ${ }^{[11]} \mathbf{2 a} \sim$ 2d were prepared according to the literature.

4.2 Typical procedure for the preparation of $\gamma$-alkynyl ketones 3

PTSA $(0.02 \mathrm{mmol})$ was added to the acetonitrile solution $(2 \mathrm{~mL})$ of propargyl alcohol $1(0.2 \mathrm{mmol})$ and enoxysilanes $2(0.8 \mathrm{mmol}, 4$ equiv. $)$ at $60{ }^{\circ} \mathrm{C}$ or room temperature. The progress of the reaction was monitored by thin layer chromatography (TLC). After $2 \mathrm{~min}$, the reaction mixture was quenched by $10 \%$ of aq. $\mathrm{Na}_{2} \mathrm{CO}_{3}$ solution, extracted with ethyl acetate. The combined organic phase was dried by $\mathrm{Na}_{2} \mathrm{SO}_{4}$, filtrated and concentrated in vacuum. The crude material was purified by flash column chromatography (silica gel, PE-EtOAc) to afford the desired product 3 .

Methyl 2-oxo-4,6-diphenylhex-5-ynoate (3a): Yield $85 \%$, light yellow oil. ${ }^{1} \mathrm{H}$ NMR ( $\left.600 \mathrm{MHz}, \mathrm{CDCl}_{3}\right) \delta: 7.46$ (d, $J=7.8 \mathrm{~Hz}, 2 \mathrm{H}), 7.43 \sim 7.40(\mathrm{~m}, 2 \mathrm{H}), 7.37 \sim 7.28(\mathrm{~m}$, $2 \mathrm{H}), 7.30 \sim 7.25(\mathrm{~m}, 4 \mathrm{H}), 4.46(\mathrm{dd}, J=6.6,8.4 \mathrm{~Hz}, 1 \mathrm{H})$, 3.84 (s, 3H), 3.49 (dd, $J=8.4,17.4 \mathrm{~Hz}, 1 \mathrm{H}), 3.34$ (dd, $J=$ $6.0,17.4 \mathrm{~Hz}, 1 \mathrm{H}) ;{ }^{13} \mathrm{C} \mathrm{NMR}\left(150 \mathrm{MHz}, \mathrm{CDCl}_{3}\right) \delta: 191.1$, 161.0, 140.1, 131.7, 128.8, 128.24, 128.16, 127.5, 127.4, 123.1, 89.4, 84.0, 53.1, 47.8, 33.2; HRMS (ESI) calcd for $\mathrm{C}_{19} \mathrm{H}_{17} \mathrm{O}_{3}[\mathrm{M}+\mathrm{H}]^{+}$293.1172, found 293.1182.

Methyl 4-(4-chlorophenyl)-2-oxo-6-phenylhex-5-ynoate (3b): Yield $82 \%$, light yellow oil. ${ }^{1} \mathrm{H}$ NMR $(600 \mathrm{MHz}$, $\left.\mathrm{CDCl}_{3}\right) \delta: 7.45 \sim 7.25(\mathrm{~m}, 9 \mathrm{H}), 4.44(\mathrm{t}, J=7.2 \mathrm{~Hz}, 1 \mathrm{H})$, $3.84(\mathrm{~s}, 3 \mathrm{H}), 3.47$ (dd, $J=7.8,18 \mathrm{~Hz}, 1 \mathrm{H}), 3.33$ (dd, $J=$ 6.6, $18 \mathrm{~Hz}, 1 \mathrm{H}) ;{ }^{13} \mathrm{C}$ NMR $\left(150 \mathrm{MHz}, \mathrm{CDCl}_{3}\right) \delta: 190.8$, $160.9,138.7,133.3,131.7,129.0,128.32,128.29,122.8$, 88.8, 84.2, 53.2, 47.5, 32.5; HRMS (ESI) calcd for $\mathrm{C}_{19} \mathrm{H}_{16} \mathrm{ClO}_{3}[\mathrm{M}+\mathrm{H}]^{+}$327.0782, found 327.0783 .

Methyl 4-(4-bromophenyl)-2-oxo-6-phenylhex-5-ynoate (3c): Yield $80 \%$, light yellow oil. ${ }^{1} \mathrm{H}$ NMR $(600 \mathrm{MHz}$, $\left.\mathrm{CDCl}_{3}\right) \delta: 7.47(\mathrm{~d}, J=9 \mathrm{~Hz}, 1 \mathrm{H}), 7.42 \sim 7.38(\mathrm{~m}, 2 \mathrm{H}), 7.34$ $(\mathrm{d}, J=8.4 \mathrm{~Hz}, 1 \mathrm{H}), 7.31 \sim 7.27(\mathrm{~m}, 3 \mathrm{H}), 4.43(\mathrm{t}, J=6.6$ Hz, 1H), 3.85 (s, 3H), 3.47 (dd, $J=7.8,17.4 \mathrm{~Hz}, 1 \mathrm{H}), 3.33$ $(\mathrm{dd}, J=6.6,17.4 \mathrm{~Hz}, 1 \mathrm{H}) ;{ }^{13} \mathrm{C}$ NMR $\left(150 \mathrm{MHz}, \mathrm{CDCl}_{3}\right) \delta$ : $190.7,160.9,139.2,131.9,131.7,129.3,128.32,128.29$, $122.8,121.3,88.7,84.3,53.2,47.5,32.6$; HRMS (ESI) calcd for $\mathrm{C}_{19} \mathrm{H}_{16} \mathrm{BrO}_{3}[\mathrm{M}+\mathrm{H}]^{+}$371.0277/373.0257, found 371.0276/373.0257.

Methyl 4-(5-bromo-2-methoxyphenyl)-2-oxo-6-phenylhex-5-ynoate (3d): Yield 78\%, colorless oil. ${ }^{1} \mathrm{H}$ NMR $(600$ $\left.\mathrm{MHz}, \mathrm{CDCl}_{3}\right) \delta: 7.76(\mathrm{~d}, J=2.4 \mathrm{~Hz}, 1 \mathrm{H}), 7.46 \sim 7.42(\mathrm{~m}$, $2 \mathrm{H}), 7.36(\mathrm{~d}, J=2.4,9.0 \mathrm{~Hz}, 1 \mathrm{H}), 7.32 \sim 7.28(\mathrm{~m}, 3 \mathrm{H})$, $6.74(\mathrm{~d}, J=8.4 \mathrm{~Hz}, 1 \mathrm{H}), 4.76(\mathrm{dd}, J=5.4,8.4 \mathrm{~Hz}, 1 \mathrm{H})$, $3.86(\mathrm{~s}, 3 \mathrm{H}), 3.81$ (s, 3H), 3.41 (dd, $J=8.4,16.8 \mathrm{~Hz}, 1 \mathrm{H})$, $3.19(\mathrm{dd}, J=5.4,16.8 \mathrm{~Hz}, 1 \mathrm{H}) ;{ }^{13} \mathrm{C}$ NMR $(150 \mathrm{MHz}$, $\left.\mathrm{CDCl}_{3}\right) \delta: 190.9,161.0,155.1,131.8,131.6,131.3,130.2$, 128.3, 128.2, 123.0, 113.2, 112.2, 88.5, 84.4, 55.6, 53.0, 45.8, 27.6; HRMS (ESI) calcd for $\mathrm{C}_{20} \mathrm{H}_{18} \mathrm{BrO}_{4}[\mathrm{M}+\mathrm{H}]^{+}$ 401.0383/403.0363, found 401.0381/403.0365.

Methyl 4-(2-methoxyphenyl)-2-oxo-6-phenylhex-5-ynoate (3e): Yield $83 \%$, light yellow oil. ${ }^{1} \mathrm{H}$ NMR $(600 \mathrm{MHz}$, $\left.\mathrm{CDCl}_{3}\right) \delta: 7.67(\mathrm{~d}, J=7.8 \mathrm{~Hz}, 1 \mathrm{H}), 7.45 \sim 7.41(\mathrm{~m}, 2 \mathrm{H})$,
$7.30 \sim 7.26(\mathrm{~m}, 4 \mathrm{H}), 6.99(\mathrm{t}, J=7.8 \mathrm{~Hz}, 1 \mathrm{H}), 6.86(\mathrm{~d}, J=$ $8.4 \mathrm{~Hz}, 1 \mathrm{H}), 4.81(\mathrm{dd}, J=5.4,8.4 \mathrm{~Hz}, 1 \mathrm{H}), 3.84(\mathrm{~s}, 3 \mathrm{H})$, $3.82(\mathrm{~s}, 3 \mathrm{H}), 3.42(\mathrm{dd}, J=8.4,16.2 \mathrm{~Hz}, 1 \mathrm{H}), 3.23(\mathrm{dd}, J=$ 5.4, $16.2 \mathrm{~Hz}, 1 \mathrm{H}) ;{ }^{13} \mathrm{C}$ NMR $\left(150 \mathrm{MHz}, \mathrm{CDCl}_{3}\right) \delta: 191.3$, $161.1,156.0,131.7,128.7,128.6,128.2,128.0,127.8$, 123.3, 120.9, 110.5, 89.4, 83.9, 55.3, 53.0, 46.1, 27.8; HRMS (ESI) calcd for $\mathrm{C}_{20} \mathrm{H}_{19} \mathrm{O}_{4}[\mathrm{M}+\mathrm{H}]^{+} 323.1278$, found 323.1279 .

Methyl 4-(4-(benzyloxy)phenyl)-2-oxo-6-phenylhex-5ynoate (3f): Yield 90\%, light yellow oil. ${ }^{1} \mathrm{H}$ NMR $(600$ $\left.\mathrm{MHz}, \mathrm{CDCl}_{3}\right) \delta: 7.44 \sim 7.26(\mathrm{~m}, 12 \mathrm{H}), 6.95(\mathrm{~d}, J=8.4 \mathrm{~Hz}$, $2 \mathrm{H}), 5.06(\mathrm{~s}, 2 \mathrm{H}), 4.42(\mathrm{dd}, J=6.6,7.8 \mathrm{~Hz}, 1 \mathrm{H}), 3.83(\mathrm{~s}$, $3 \mathrm{H}), 3.45$ (dd, $J=7.8,17.4 \mathrm{~Hz}, 1 \mathrm{H}), 3.32$ (dd, $J=6.6,17.4$ $\mathrm{Hz}, 1 \mathrm{H}) ;{ }^{13} \mathrm{C}$ NMR $\left(150 \mathrm{MHz}, \mathrm{CDCl}_{3}\right) \delta: 191.2,161.0$, $158.1,137.0,132.5,131.7,128.63,128.61,128.2,128.1$, $128.0,127.5,123.1,115.1,89.6,83.8,70.1,53.1,47.9$, 32.4; HRMS (ESI) calcd for $\mathrm{C}_{26} \mathrm{H}_{23} \mathrm{O}_{4}[\mathrm{M}+\mathrm{H}]^{+}$ 399.1591 , found 399.1592 .

Methyl 4-(4-(benzyloxy)phenyl)-2-oxoundec-5-ynoate (3g): Yield $88 \%$, yellow oil. ${ }^{1} \mathrm{H}$ NMR $\left(600 \mathrm{MHz}, \mathrm{CDCl}_{3}\right)$ $\delta: 7.42(\mathrm{~d}, J=7.8 \mathrm{~Hz}, 2 \mathrm{H}), 7.38(\mathrm{t}, J=7.8 \mathrm{~Hz}, 2 \mathrm{H}), 7.34 \sim$ $7.28(\mathrm{~m}, 3 \mathrm{H}), 6.92(\mathrm{~d}, J=9.0 \mathrm{~Hz}, 2 \mathrm{H}), 5.04(\mathrm{~s}, 2 \mathrm{H}), 4.20 \sim$ $4.15(\mathrm{~m}, 1 \mathrm{H}), 3.84(\mathrm{~s}, 3 \mathrm{H}), 3.30$ (dd, $J=8.4,16.8 \mathrm{~Hz}, 1 \mathrm{H})$, $3.18(\mathrm{dd}, J=6.0,16.8 \mathrm{~Hz}, 1 \mathrm{H}), 2.18 \sim 2.14(\mathrm{~m}, 2 \mathrm{H})$, $1.51 \sim 1.45(\mathrm{~m}, 2 \mathrm{H}), 1.38 \sim 1.25(\mathrm{~m}, 4 \mathrm{H}), 0.89(\mathrm{t}, J=7.2$ $\mathrm{Hz}, 3 \mathrm{H}) ;{ }^{13} \mathrm{C}$ NMR $\left(150 \mathrm{MHz}, \mathrm{CDCl}_{3}\right) \delta: 191.4,161.0$, $157.9,137.0,133.2,128.6,128.5,128.0,127.5,115.0$, 84.3, 80.0, 70.1, 53.0, 48.2, 32.0, 31.0, 28.5, 22.2, 18.7, 14.0; HRMS (ESI) calcd for $\mathrm{C}_{25} \mathrm{H}_{29} \mathrm{O}_{4}[\mathrm{M}+\mathrm{H}]^{+}$ 393.2060, found 393.2059.

Methyl 4-(naphthalen-2-yl)-2-oxo-6-phenylhex-5-ynoate (3h): Yield 91\%, light yellow oil. ${ }^{1} \mathrm{H}$ NMR $(600 \mathrm{MHz}$, $\left.\mathrm{CDCl}_{3}\right) \delta: 7.91(\mathrm{~s}, 1 \mathrm{H}), 7.86 \sim 7.80(\mathrm{~m}, 3 \mathrm{H}), 7.58(\mathrm{dd}, J=$ $1.8,8.4 \mathrm{~Hz}, 1 \mathrm{H}), 7.50 \sim 7.42(\mathrm{~m}, 4 \mathrm{H}), 7.31 \sim 7.28(\mathrm{~m}, 3 \mathrm{H})$, $4.64(\mathrm{dd}, J=6.6,7.8 \mathrm{~Hz}, 1 \mathrm{H}), 3.82(\mathrm{~s}, 3 \mathrm{H}), 3.57$ (dd, $J=$ $8.4,17.4 \mathrm{~Hz}, 1 \mathrm{H}), 3.44(\mathrm{dd}, J=6.6,17.4 \mathrm{~Hz}, 1 \mathrm{H}) ;{ }^{13} \mathrm{C}$ NMR $\left(150 \mathrm{MHz}, \mathrm{CDCl}_{3}\right) \delta: 191.0,161.0,137.5,133.5$, $132.7,131.8,128.7,128.3,128.2,127.9,127.7,126.4$, 126.3, 126.1, 125.5, 123.1, 89.3, 84.2, 53.1, 47.6, 33.3; HRMS (ESI) calcd for $\mathrm{C}_{23} \mathrm{H}_{19} \mathrm{O}_{3}[\mathrm{M}+\mathrm{H}]^{+}$343.1329, found 343.1328 .

Methyl 4-(3-fluoro-4-methoxyphenyl)-2-oxo-6-phenylhex-5-ynoate (3i): Yield 88\%, light yellow oil. ${ }^{1} \mathrm{H}$ NMR $\left(600 \mathrm{MHz}, \mathrm{CDCl}_{3}\right) \delta: 7.42 \sim 7.39(\mathrm{~m}, 2 \mathrm{H}), 7.31 \sim 7.28(\mathrm{~m}$, $3 \mathrm{H}), 7.22(\mathrm{dd}, J=1.8,12.0 \mathrm{~Hz}, 1 \mathrm{H}), 7.15(\mathrm{~d}, J=8.4 \mathrm{~Hz}$, $1 \mathrm{H}), 6.92(\mathrm{t}, J=8.4 \mathrm{~Hz}, 1 \mathrm{H}), 4.41(\mathrm{t}, J=7.8 \mathrm{~Hz}, 1 \mathrm{H}), 3.88$ (s, 3H), 3.85 (s, 3H), 3.45 (dd, $J=7.8,17.4 \mathrm{~Hz}, 1 \mathrm{H}), 3.32$ (dd, $J=6.6,17.4 \mathrm{~Hz}, 1 \mathrm{H}) ;{ }^{13} \mathrm{C}$ NMR $\left(150 \mathrm{MHz}, \mathrm{CDCl}_{3}\right) \delta$ : $190.9,160.9,152.4(\mathrm{~d}, J=244.95 \mathrm{~Hz}), 146.9$ (d, $J=10.8$ Hz), 133.1 (d, $J=5.4 \mathrm{~Hz}), 131.7,128.3,123.2(\mathrm{~d}, J=3.15$ $\mathrm{Hz}), 122.9,115.4$ (d, $J=19.5 \mathrm{~Hz}), 113.6,89.0,84.1,56.4$, 53.1, 47.7, 32.2; HRMS (ESI) calcd for $\mathrm{C}_{20} \mathrm{H}_{18} \mathrm{FO}_{4}[\mathrm{M}+$ $\mathrm{H}]^{+}$341.1184, found 341.1184.

Methyl 4-(3,4-dimethylphenyl)-2-oxo-6-phenylhex-5ynoate $(\mathbf{3 j})$ : Yield $92 \%$, light yellow oil. ${ }^{1} \mathrm{H}$ NMR $(600$ $\left.\mathrm{MHz}, \mathrm{CDCl}_{3}\right) \delta: 7.42 \sim 7.38(\mathrm{~m}, 2 \mathrm{H}), 7.29 \sim 7.26(\mathrm{~m}, 3 \mathrm{H})$, $7.21(\mathrm{~s}, 1 \mathrm{H}), 7.19 \sim 7.17(\mathrm{~m}, 1 \mathrm{H}), 7.11(\mathrm{~d}, J=7.2 \mathrm{~Hz}, 1 \mathrm{H})$, 
4.40 (dd, $J=6.6,8.4 \mathrm{~Hz}, 1 \mathrm{H}), 3.84$ (s, 3H), 3.47 (dd, $J=$ 8.4, 17.4 Hz, 1H), 3.31 (dd, $J=6.6,17.4 \mathrm{~Hz}, 1 \mathrm{H}), 2.27$ (s, $3 \mathrm{H}), 2.24(\mathrm{~s}, 3 \mathrm{H}) ;{ }^{13} \mathrm{C}$ NMR $\left(150 \mathrm{MHz}, \mathrm{CDCl}_{3}\right) \delta: 191.2$, $161.0,137.6,137.1,135.7,131.7,130.0,128.7,128.2$, 128.0, 124.8, 123.2, 89.7, 83.7, 53.1, 47.8, 32.8, 19.8, 19.4; HRMS (ESI) calcd for $\mathrm{C}_{21} \mathrm{H}_{21} \mathrm{O}_{3}[\mathrm{M}+\mathrm{H}]^{+}$321.1485, found 321.1485 .

Methyl 4-(3,4-dimethoxyphenyl)-2-oxo-6-phenylhex-5ynoate (3k): Yield 95\%, light yellow oil. ${ }^{1} \mathrm{H}$ NMR (600 $\left.\mathrm{MHz}, \mathrm{CDCl}_{3}\right) \delta: 7.43 \sim 7.38(\mathrm{~m}, 2 \mathrm{H}), 7.31 \sim 7.27(\mathrm{~m}, 3 \mathrm{H})$, $7.02 \sim 6.97(\mathrm{~m}, 2 \mathrm{H}), 6.84(\mathrm{~d}, J=7.8 \mathrm{~Hz}, 1 \mathrm{H}), 4.42(\mathrm{t}, J=$ $7.2 \mathrm{~Hz}, 1 \mathrm{H}), 3.91$ (s, 3H), 3.87 (s, 3H), 3.85 (s, 3H), 3.47 (dd, $J=7.8,17.4 \mathrm{~Hz}, 1 \mathrm{H}), 3.34$ (dd, $J=6.6,17.4 \mathrm{~Hz}, 1 \mathrm{H})$; ${ }^{13} \mathrm{C}$ NMR $\left(150 \mathrm{MHz}, \mathrm{CDCl}_{3}\right) \delta: 191.2,161.0,149.1,148.3$, $132.7,131.7,128.3,128.2,123.1,119.6,111.4,110.8$, 89.6, 83.9, 56.0, 55.9, 53.1, 47.8, 32.8; HRMS (ESI) calcd for $\mathrm{C}_{21} \mathrm{H}_{21} \mathrm{O}_{5}[\mathrm{M}+\mathrm{H}]^{+}$353.1384, found 353.1387.

2-(1-(3,4-Dimethoxyphenyl)-3-phenylprop-2-yn-1-yl)cyclohexan-1-one (3l-1): one of the two diastereomers, Yield 54\%, colorless oil. ${ }^{1} \mathrm{H}$ NMR $\left(600 \mathrm{MHz}, \mathrm{CDCl}_{3}\right) \delta$ : $7.42 \sim 7.39(\mathrm{~m}, 2 \mathrm{H}), 7.29 \sim 7.25(\mathrm{~m}, 3 \mathrm{H}), 7.01(\mathrm{~d}, J=1.8$ $\mathrm{Hz}, 1 \mathrm{H}), 6.97(\mathrm{dd}, J=1.8,8.4 \mathrm{~Hz}, 1 \mathrm{H}), 6.82(\mathrm{~d}, J=8.4 \mathrm{~Hz}$, $1 \mathrm{H}), 4.37$ (d, $J=6.6 \mathrm{~Hz}, 1 \mathrm{H}), 3.89$ (s, 3H), 3.87 (s, 3H), $2.94 \sim 2.84(\mathrm{~m}, 1 \mathrm{H}), 2.50 \sim 2.48(\mathrm{~m}, 1 \mathrm{H}), 2.40 \sim 2.37(\mathrm{~m}$, 1H), $2.05 \sim 1.60(\mathrm{~m}, 6 \mathrm{H}) ;{ }^{13} \mathrm{C}$ NMR $\left(150 \mathrm{MHz}, \mathrm{CDCl}_{3}\right) \delta$ : $210.5,148.8,148.0,131.7,131.5,128.2,127.8,123.6$, $121.1,112.2,110.9,91.4,82.7,57.4,55.94,55.91,42.1$, 36.6, 30.7, 27.7, 24.6; HRMS (ESI) calcd for $\mathrm{C}_{23} \mathrm{H}_{25} \mathrm{O}_{3}$ $[\mathrm{M}+\mathrm{H}]^{+}$349.1798, found 349.1787.

2-(1-(3,4-Dimethoxyphenyl)-3-phenylprop-2-yn-1-yl)cyclohexan-1-one (3l-2): one of the two diastereomers: Yield 39\%, colorless oil. ${ }^{1} \mathrm{H}$ NMR $\left(600 \mathrm{MHz}, \mathrm{CDCl}_{3}\right) \delta$ : $7.45 \sim 7.41(\mathrm{~m}, 2 \mathrm{H}), 7.31 \sim 7.27(\mathrm{~m}, 3 \mathrm{H}), 6.99(\mathrm{dd}, J=1.8$, $7.8 \mathrm{~Hz}, 1 \mathrm{H}), 6.97$ (d, $J=1.8 \mathrm{~Hz}, 1 \mathrm{H}), 6.83(\mathrm{~d}, J=8.4 \mathrm{~Hz}$, $1 \mathrm{H}), 4.60(\mathrm{~d}, J=4.8 \mathrm{~Hz}, 1 \mathrm{H}), 3.90(\mathrm{~s}, 3 \mathrm{H}), 3.87$ (s, 3H), $2.63 \sim 2.57(\mathrm{~m}, 1 \mathrm{H}), 2.52 \sim 2.46(\mathrm{~m}, 1 \mathrm{H}), 2.30(\mathrm{dt}, J=6.0$, $13.2 \mathrm{~Hz}, 1 \mathrm{H}), 2.22 \sim 2.17(\mathrm{~m}, 1 \mathrm{H}), 2.09 \sim 2.03(\mathrm{~m}, 1 \mathrm{H})$, $1.96 \sim 1.82(\mathrm{~m}, 2 \mathrm{H}), 1.76 \sim 1.56(\mathrm{~m}, 2 \mathrm{H}) ;{ }^{13} \mathrm{C}$ NMR $(150$ $\left.\mathrm{MHz}, \mathrm{CDCl}_{3}\right) \delta: 210.1,148.8,147.9,133.2,131.7,128.2$, $127.8,123.7,120.1,111.5,111.1,89.3,84.8,57.1,55.9$, 42.1, 36.7, 29.5, 27.4, 24.9; HRMS (ESI) calcd for $\mathrm{C}_{23} \mathrm{H}_{25} \mathrm{O}_{3}[\mathrm{M}+\mathrm{H}]^{+}$349.1798, found 349.1785.

2-(1-(4-(Benzyloxy)phenyl)-3-phenylprop-2-yn-1-yl)cyclohexan-1-one (3m-1): one of the two diastereomers, Yield 45\%, colorless oil. ${ }^{1} \mathrm{H}$ NMR $\left(600 \mathrm{MHz}, \mathrm{CDCl}_{3}\right) \delta$ : $7.45 \sim 7.30(\mathrm{~m}, 9 \mathrm{H}), 7.28 \sim 7.24(\mathrm{~m}, 3 \mathrm{H}), 6.93(\mathrm{~d}, J=9.0$ $\mathrm{Hz}, 2 \mathrm{H}), 5.05(\mathrm{~s}, 2 \mathrm{H}), 4.36(\mathrm{~d}, J=6.6 \mathrm{~Hz}, 1 \mathrm{H}), 2.86 \sim 2.80$ $(\mathrm{m}, 1 \mathrm{H}), 2.48 \sim 2.43(\mathrm{~m}, 1 \mathrm{H}), 2.39 \sim 2.32(\mathrm{~m}, 1 \mathrm{H}), 2.05 \sim$ $1.80(\mathrm{~m}, 4 \mathrm{H}), 1.68 \sim 1.58(\mathrm{~m}, 2 \mathrm{H}) ;{ }^{13} \mathrm{C}$ NMR $(150 \mathrm{MHz}$, $\left.\mathrm{CDCl}_{3}\right) \delta: 210.4,157.9,137.1,131.7,131.4,130.0,128.6$ $128.1,128.0,127.8,127.5,123.7,114.6,91.5,82.6,70.1$, 57.3, 42.1, 36.3, 30.8, 27.7, 24.7; HRMS (ESI) calcd for $\mathrm{C}_{28} \mathrm{H}_{27} \mathrm{O}_{2}[\mathrm{M}+\mathrm{H}]^{+}$395.2006, found 395.2006.

2-(1-(4-(Benzyloxy)phenyl)-3-phenylprop-2-yn-1-yl)cyclohexan-1-one (3m-2): one of the two diastereomers, Yield 50\%, colorless oil. ${ }^{1} \mathrm{H}$ NMR $\left(600 \mathrm{MHz}, \mathrm{CDCl}_{3}\right) \delta$ : $7.45 \sim 7.26(\mathrm{~m}, 12 \mathrm{H}), 6.94(\mathrm{~d}, J=8.4 \mathrm{~Hz}, 2 \mathrm{H}), 5.05(\mathrm{~s}$,
2H), 4.59 (d, J=4.8 Hz, 1H), $2.61 \sim 2.56(\mathrm{~m}, 1 \mathrm{H}), 2.50 \sim$ $2.45(\mathrm{~m}, 1 \mathrm{H}), 2.32 \sim 2.24(\mathrm{dt}, J=6.6,13.8 \mathrm{~Hz}, 2 \mathrm{H}), 2.22 \sim$ $2.15(\mathrm{~m}, 1 \mathrm{H}), 2.08 \sim 2.02(\mathrm{~m}, 1 \mathrm{H}), 1.95 \sim 1.81(\mathrm{~m}, 2 \mathrm{H})$, $1.76 \sim 1.66(\mathrm{~m}, 1 \mathrm{H}), 1.63 \sim 1.55(\mathrm{~m}, 1 \mathrm{H}) ;{ }^{13} \mathrm{C}$ NMR $(150$ $\left.\mathrm{MHz}, \mathrm{CDCl}_{3}\right) \delta: 210.1,157.8,137.1,133.0,131.7,129.1$, 128.6, 128.2, 128.0, 127.8, 127.5, 123.7, 114.8, 89.4, 84.7, 70.1, 57.1, 42.2, 36.3, 29.4, 27.4, 24.9; HRMS (ESI) calcd for $\mathrm{C}_{28} \mathrm{H}_{27} \mathrm{O}_{2}[\mathrm{M}+\mathrm{H}]^{+}$395.2006, found 395.2004.

4-(3,4-Dimethoxyphenyl)-6-phenylhex-5-yn-2-one (3n): Yield 79\%, light yellow oil. ${ }^{1} \mathrm{H}$ NMR $\left(600 \mathrm{MHz}, \mathrm{CDCl}_{3}\right)$ $\delta: 7.43 \sim 7.39(\mathrm{~m}, 2 \mathrm{H}), 7.31 \sim 7.27(\mathrm{~m}, 3 \mathrm{H}), 7.01 \sim 6.97$ $(\mathrm{m}, 2 \mathrm{H}), 6.84(\mathrm{~d}, J=8.4 \mathrm{~Hz}, 1 \mathrm{H}), 4.37(\mathrm{t}, J=7.2 \mathrm{~Hz}, 1 \mathrm{H})$, 3.90 (s, 3H), 3.87 (s, 3H), 3.06 (dd, $J=7.8,16.2 \mathrm{~Hz}, 1 \mathrm{H})$, $2.89(\mathrm{dd}, J=6.6,16.2 \mathrm{~Hz}, 1 \mathrm{H}), 2.18(\mathrm{~s}, 3 \mathrm{H}) ;{ }^{13} \mathrm{C} \mathrm{NMR}$ $\left(150 \mathrm{MHz}, \mathrm{CDCl}_{3}\right) \delta: 206.0,149.1,148.1,133.5,131.6$, $128.3,128.0,123.3,119.4,111.3,110.8,90.6,83.2,56.0$, 55.9, 52.1, 33.2; HRMS (ESI) calcd for $\mathrm{C}_{20} \mathrm{H}_{21} \mathrm{O}_{3}[\mathrm{M}+$ $\mathrm{H}]^{+}$309.1485, found 309.1486.

3-(3,4-Dimethoxyphenyl)-1,5-diphenylpent-4-yn-1-one (3o): Yield 85\%, light yellow oil. ${ }^{1} \mathrm{H}$ NMR $(600 \mathrm{MHz}$, $\left.\mathrm{CDCl}_{3}\right) \delta: 7.97(\mathrm{~d}, J=7.8 \mathrm{~Hz}, 2 \mathrm{H}), 7.56(\mathrm{t}, J=7.8 \mathrm{~Hz}, 1 \mathrm{H})$, $7.46(\mathrm{t}, J=7.8 \mathrm{~Hz}, 2 \mathrm{H}), 7.37 \sim 7.34(\mathrm{~m}, 2 \mathrm{H}), 7.28 \sim 7.24$ $(\mathrm{m}, 3 \mathrm{H}), 7.08 \sim 7.03(\mathrm{~m}, 2 \mathrm{H}), 6.84(\mathrm{~d}, J=7.8 \mathrm{~Hz}, 1 \mathrm{H})$, $4.60(\mathrm{t}, J=6.6 \mathrm{~Hz}, 1 \mathrm{H}), 3.90(\mathrm{~s}, 3 \mathrm{H}), 3.86(\mathrm{~s}, 3 \mathrm{H}), 3.64$ (dd, $J=7.8,16.8 \mathrm{~Hz}, 1 \mathrm{H}), 3.41$ (dd, $J=6.6,16.8 \mathrm{~Hz}, 1 \mathrm{H})$; ${ }^{13} \mathrm{C} \mathrm{NMR}\left(150 \mathrm{MHz}, \mathrm{CDCl}_{3}\right) \delta: 197.4,149.0,148.1,137.0$, $133.8,133.3,131.6,128.7,128.24,128.19,127.9,123.4$, 119.6, 111.3, 111.0, 91.0, 83.3, 56.0, 55.9, 47.4, 33.4; HRMS (ESI) calcd for $\mathrm{C}_{25} \mathrm{H}_{23} \mathrm{O}_{3}[\mathrm{M}+\mathrm{H}]^{+}$371.1642, found 371.1642 .

Supporting Information ${ }^{1} \mathrm{H}$ NMR and ${ }^{13} \mathrm{C}$ NMR spectra of compounds $\mathbf{3 a} \sim \mathbf{3 o}$. The Supporting Information is available free of charge via the Internet at http://siocjournal.cn/.

\section{References}

[1] (a) Roy, R.; Saha, S. RSC Adv. 2018, 8, 31129. (b) Zhu, Y.; Sun, L.; Lu, P.; Wang, Y. ACS Catal. 2014, 4, 1911. (c) Ayers, B. J.; Chan, P. W. H. Synlett 2015, 26, 1305.

(d) Zhang, L.; Fang, G.; Kumar, R. K.; Bi, X. Synthesis 2015, 47, 2317.

(e) Zhang, X. X.; Lv, C.; Li, P.; Fu, B. Chin. J. Org. Chem. 2016, 36, 1287 (in Chinese).

(张小祥, 吕昌, 李萍, 付博, 姚薇薇, 有机化学, 2016, 36, 1287.) (f) Lu, F. D.; Jiang, X.; Lu, L. Q.; Xiao, W. J. Acta Chim. Sinica 2019, 77, 803 (in Chinese).

(路福东, 姜炫, 陆良秋, 肖文精, 化学学报, 2019, 77, 803.)

[2] (a) Srihari P.; Bhunia D. C.; Sreedhar, P.; Mandal S. S.; Reddy J. S. S.; Yadav J. S. Tetrahedron Lett. 2007, 48, 8120.

(b) Luzung, M.; Toste, F. J. Am. Chem. Soc. 2003, 125, 15760.

(c) Georgy, M.; Boucard, V.; Campagne, J. J. Am. Chem. Soc. 2005, 127, 14180.

[3] Sanz, R.; Miguel, D.; Martínez, A.; Álvarez-Gutiérrez, J.; Rodríguez, F. Org. Lett. 2007, 9, 727.

[4] Mitsuhiro, Y.; Tokutaro, O.; Saki, M.; Teruhisa, Y.; Arisa, S. Org. Lett. 2008, 10, 4251.

[5] Matsuda, I.; Komori, K.; Itoh, K. J. Am. Chem. Soc. 2002, 124, 9072.

[6] Zhan, Z.; Wang, S.; Cai, X.; Liu, H.; Yu, J.; Cui, Y. Adv. Synth. 
Catal. 2007, 349, 2097.

[7] Zhan, Z.; Cai, X.; Wang, S.; Yu, J.; Liu, H.; Cui, Y. J. Org. Chem. 2007, 72, 9838 .

[8] Lin, M.; Hao, L.; Ma, R.; Zhan, Z. Synlett 2010, 2345.

[9] Naveen, N.; Koppolu, S.; Balamurugan, R. Adv. Synth. Catal. 2015, $357,1463$.

[10] (a) Zhu, H.; Ji, K.; Yang, F.; Wang, L.; Zhao, S.; Ali, S.; Liu, X.; Liang, Y. Org. Lett. 2011, 13, 684.

(b) Zhang, S.; Liu, H. Chin. J. Org. Chem. 2020, 40, 1257 (in Chinese).

(张顺吉, 刘会丽, 有机化学, 2020, 40, 1257.)

(c) Michael, R., L.; Dean, T. J. Am. Chem. Soc. 2003, 125, 15760.

(d) Zhou, J.; Zhang, G.; Zou, J.; Zhang, W. Eur. J. Org. Chem.
2011, 19, 3412.

(e) Lin, C.; Duh, T.; Lu, W.; Lee, J.; Lee, C.; Chen, C.; Wu, M. J. Chin. Chem. Soc. 2004, 51, 183.

(f) Zhou, C.; Dubrovsky, A.; Larock, R. J. Org. Chem. 2006, 71, 1626.

(g) Tyrrell, E.; Tesfa, K.; Mann, A.; Singh, K. Synthesis, 2007, 1491.

(h) Li, Z.; Pu, L. Org. Lett. 2004, 6, 1065.

[11] (a) Cazeau, P.; Duboudin, F.; Moulines, F.; Babot, O.; Dunogues, J. Tetrahedron 1986, 43, 2075.

(b) Leijondahl, K.; Boren, L.; Braun, R.; Baeckvall, J. Org. Lett. 2008, 10, 2027.

(Fan, Y.) 